Supporting Information

\title{
Study of Charge Distributions and Electrical Properties in GaAs/AIGaAs Single Quantum Well/Nanowire Heterostructures
}

Chen Li, ${ }^{1}$ Yongfa Cheng, ${ }^{1}$ Bang Li, ${ }^{2}$ Feng Cheng,,${ }^{1}$ Luying Li,, * Tianyu Qi, ${ }^{1}$

Shuangfeng Jia, ${ }^{3}$ Xin Yan, ${ }^{2 *}$ Xia Zhang, ${ }^{2}$ Jianbo Wang, ${ }^{3}$ and Yihua Gao ${ }^{1}$

${ }^{1}$ Center for Nanoscale Characterization \& Devices, Wuhan National Laboratory for Optoelectronics and School of Physics, Huazhong University of Science and Technology, Luoyu Road 1037, Wuhan, 430074, China.

${ }^{2}$ State Key Laboratory of Information Photonics and Optical Communications, Beijing University of Posts and Telecommunications, Beijing 100876, China.

${ }^{3}$ School of Physics and Technology, Center for Electron Microscopy, MOE Key

Laboratory of Artificial Micro- and Nano-Structures and the Institute for Advanced Studies, Wuhan University, Wuhan, 430072, China.

*Corresponding authors. Email: luying.li@hust.edu.cn, xyan@bupt.edu.cn 
S1. Quantitative analysis of the EDS spectra.

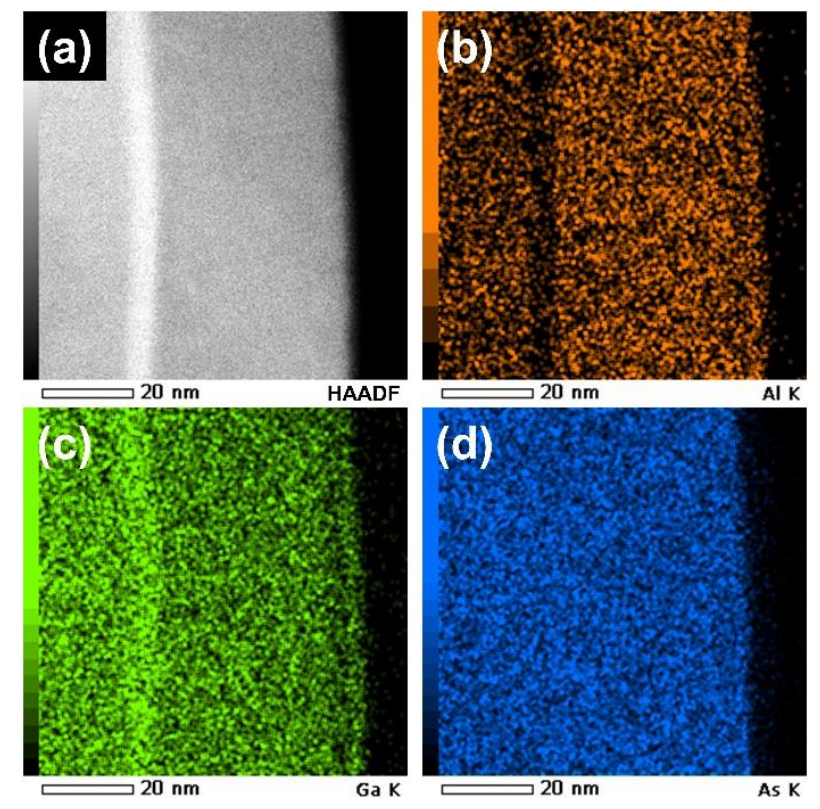

Figure S1. EDS analysis of the nanowire cross section. (a) The cross-sectional view HAADF-STEM image. (b-d) The corresponding elemental maps of the cross section, indicating distributions of $\mathrm{Al}$ (orange), Ga (green), and As (blue).

Table S1. EDS quantitative analysis of the shell region of the cross-sectional sample in Figure 3(a).

\begin{tabular}{|l|l|l|l|l|l|}
\hline Element & Weight \% & Atomic \% & Uncert. \% & Correction & k-Factor \\
\hline Al (K) & 7.84 & 18.76 & 0.28 & 0.92 & 1.030 \\
\hline Ga (K) & 28.58 & 26.46 & 0.70 & 0.99 & 1.930 \\
\hline As (K) & 63.57 & 54.76 & 0.96 & 0.99 & 2.225 \\
\hline
\end{tabular}

Table S2. EDS quantitative analysis of the core region of the cross-sectional sample 
in Figure 3(a).

\begin{tabular}{|l|l|l|l|l|l|}
\hline Element & Weight \% & Atomic \% & Uncert. \% & Correction & k-Factor \\
\hline Al (K) & 4.11 & 10.33 & 0.11 & 0.92 & 1.030 \\
\hline Ga (K) & 43.77 & 42.54 & 0.37 & 0.99 & 1.930 \\
\hline As (K) & 52.10 & 47.12 & 0.46 & 0.99 & 2.225 \\
\hline
\end{tabular}

The concentrations of aluminum from the shell and core of GaAs/AlGaAs Single Quantum Well/ Nanowire are calculated based on Table S1 and Table S2, respectively. According to Table $\mathrm{S} 1$, the atomic ratio between $\mathrm{Ga}$ and $\mathrm{Al}$ in the shell is calculated to be about 1.4, which means the aluminum concentration of the shell ( $x$ in $\mathrm{Al}_{x} \mathrm{Ga}_{1-x} \mathrm{As}$ ) is $\sim 0.4$. Similarly, the concentration of aluminum in the core can be obtained from Table S2, which is calculated $\left(x\right.$ in $\mathrm{Al}_{x} \mathrm{Ga}_{1-x} \mathrm{As}$ ) to be $\sim 0.2$.

\section{S2. The standard process of obtaining thickness image.}

The standard electron holography reconstruction is conducted using a script embedded in Digital Micrograph. It is reported that the amplitude image can be converted into an image that is proportional to $t / \lambda_{i}$ (as shown in Equation $\left.(\mathrm{S} 1)\right)^{1}$. According to Equation (S1), the projected sample thickness is acquired based on the corresponding amplitude image applying the inelastic mean free path (IMFP) of specific material.

$$
\frac{\mathrm{t}}{\lambda_{\mathrm{i}}}=-2 \ln \left(\mathrm{A}_{\mathrm{o}} / \mathrm{A}_{\mathrm{r}}\right)
$$

where $t$ is the thickness of the sample, $\lambda_{i}$ is the IMFP, and $A_{0}, A_{r}$ are amplitude of 
the modulated object wave and amplitude of the reference wave, respectively.

By using above method, the exact thickness of $\mathrm{Al}_{0.2} \mathrm{Ga}_{0.8} \mathrm{As}$ (core) is obtained applying its IMFP. The thicknesses of the GaAs QW and $\mathrm{Al}_{0.4} \mathrm{Ga}_{0.6} \mathrm{As}$ (shell) can be obtained through further treatments as shown below.

Since the value of $\ln \left(A_{o} / A_{r}\right)$ derived from the same amplitude image is equal for all three layers, namely the values of $t / \lambda_{i}$ for different compounds are the same (as shown in Equation (S2)), the actual thicknesses of the shell and QW regions can be abstracted from Equation (S3) and Equation (S4), respectively.

$$
\begin{aligned}
-2 \ln \left(\mathrm{A}_{\mathrm{o}} / \mathrm{A}_{\mathrm{r}}\right) & =\frac{\mathrm{t}_{\text {(shell) }}}{\lambda_{\text {(shell) }}}=\frac{\mathrm{t}_{(\mathrm{QW})}}{\lambda_{(\mathrm{QW})}}=\frac{\mathrm{t}_{\text {(core) }}}{\lambda_{\text {(core) }}} \\
\mathrm{t}_{(\text {shell })} & =\frac{\lambda_{\text {(shell) }}}{\lambda_{\text {(core) }}} \mathrm{t}_{\text {(core })} \\
\mathrm{t}_{(\mathrm{QW})} & =\frac{\lambda_{(\mathrm{QW})}}{\lambda_{\text {(core) }}} \mathrm{t}_{\text {(core) }}
\end{aligned}
$$

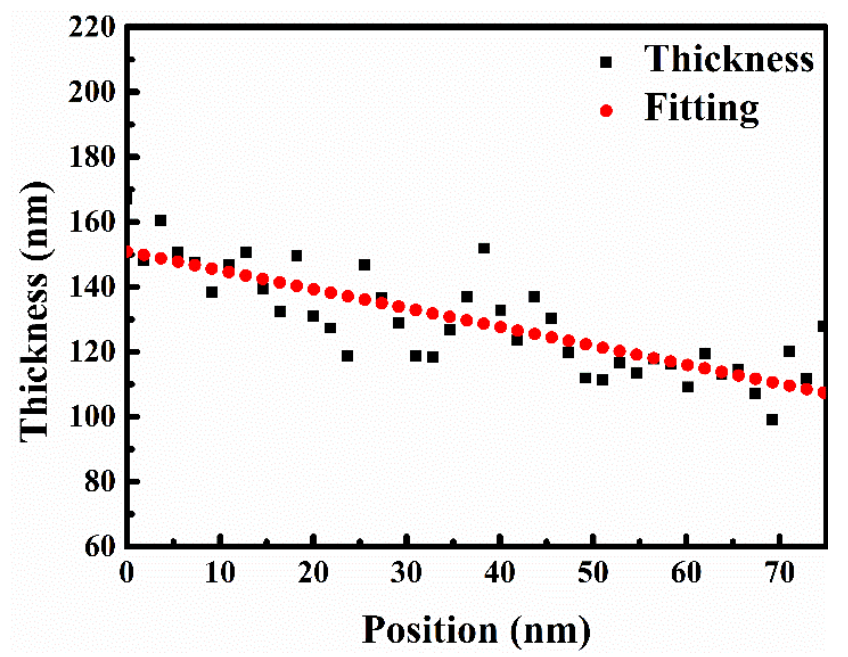

Figure S2. Projected thickness profile of the arrows-marked white box region (across the GaAs QW) obtained from Figure 4c, which is linearly fitted to the red dotted line.

\section{S3. Photoluminescence measurements.}




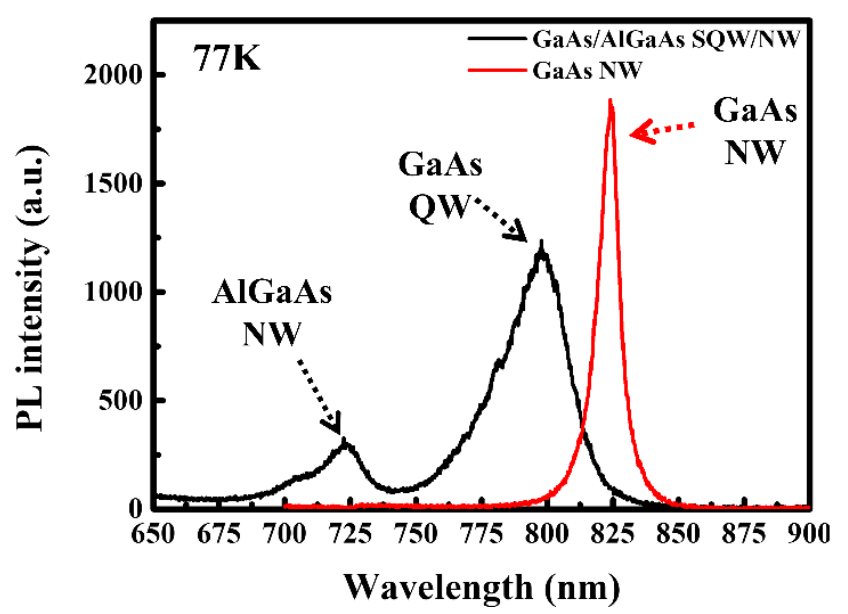

Figure S3. Low-temperature photoluminescence spectra of GaAs/AlGaAs SQW/NW (black) and single GaAs NW (red), respectively.

\section{S4. First-Principles Calculations.}

The structural relaxation and electronic calculations are performed using the allelectron double numerical atomic orbital plus polarization (DNP) basis by first principles calculations in the context of density functional theory as implemented in the $\mathrm{DMOL}^{3}$ code..$^{2,3}$ The exchange-correlation interaction is treated within the generalized gradient approximation (GGA) to the Perdew-Burke-Ernzerhof (PBE). ${ }^{4}$ The longrange van der Waals interactions are taken into account using the DFT-D2 dispersion correction proposed by Grimme. ${ }^{5}$ Besides, the atomic positions and cell vectors are relaxed until the energy, maximum force and maximum displacement are less than 1.0 $\times 10^{-5} \mathrm{Ha}, 0.002 \mathrm{Ha} \AA^{-1}$, and $0.005 \AA$, respectively. The vacuum spaces of no less than $15 \AA$ are used to avoid the interaction between the adjacent images for the single-layer structure. The reciprocal space is sampled with dense grids of $16 \times 16 \times 1 \mathrm{k}$-points in the Brillouin Zone (BZ) for the structural optimizations and electronic calculations. 
To obtain a thorough knowledge of the electrical properties of GaAs QW, we mimic the geometric structure of the GaAs QW and discuss the related electric properties. The graphene-like 2D structure of GaAs is used due to the nanoscale thickness of GaAs QW, which is cleaved perpendicular to the [111] direction of bulk GaAs, and is in good agreement with the aforementioned growth direction of its crystal. For the $\mathrm{Al}_{0.4} \mathrm{Ga}_{0.6} \mathrm{As}$ and $\mathrm{Al}_{0.2} \mathrm{Ga}_{0.8} \mathrm{As}$ layers, the aluminum atoms are introduced into the GaAs system by substitutional doping, which are combined with the horizontal atomic scale GaAs QW structure to simulate the $\mathrm{Al}_{0.4} \mathrm{Ga}_{0.6} \mathrm{As}-\mathrm{GaAs}-\mathrm{Al}_{0.2} \mathrm{Ga}_{0.8} \mathrm{As}$ Nanostructure. According to the calculations of the electronic structure, it is found that the impurity atoms cause obvious expansion of the band gap, which is increased from 1.49 $\mathrm{eV}$ to $1.78 \mathrm{eV}$ for $\mathrm{Al}_{0.4} \mathrm{Ga}_{0.6} \mathrm{As}$. Interestingly, the band structures of the impurity systems transfer to direct band gap from the indirect band gap, and the GaAs QW within the $\mathrm{Al}_{0.4} \mathrm{Ga}_{0.6} \mathrm{As}-\mathrm{GaAs}-\mathrm{Al}_{0.2} \mathrm{Ga}{ }_{0.8} \mathrm{As}$ structures presents an even larger direct band gap of $1.81 \mathrm{eV}$. For a better demonstration of the mechanism of interaction in the electric properties of the GaAs QW structure, the $\mathrm{Al}_{0.4} \mathrm{Ga}_{0.6} \mathrm{As}-\mathrm{GaAs}$ interface is selected as the research object, and the corresponding calculation of the charge density difference (CDD) is performed based on the following formula:

$$
\Delta \rho_{\text {interface }}=\rho_{\text {sum }}-\rho_{\mathrm{GaAs}}-\rho_{\mathrm{Al}_{0.4} \mathrm{Ga}_{0.6} \mathrm{As}}
$$

where $\rho_{\text {sum }}, \rho_{\mathrm{GaAs}}$, and $\rho_{\mathrm{Al}_{0.4} \mathrm{Ga}_{0.6} \mathrm{As}}$ are the total charge densities of the $\mathrm{Al}_{0.4} \mathrm{Ga}_{0.6} \mathrm{As}$ GaAs interface, pristine GaAs and isolated $\mathrm{Al}_{0.4} \mathrm{Ga}_{0.6} \mathrm{As}$, respectively.

\section{REFERENCES}


1 McCartney, M. R.; Gajdardziska-Josifovska, M. Absolute measurement of normalized thickness, $\mathrm{t} / \lambda_{\mathrm{i}}$, from off-axis electron holography. Ultramicroscopy 1994, $53,283-289$.

2 Delley, B. From molecules to solids with the DMol3 approach. J. Chem. Phys. 2000, $113,7756-7764$.

3 Cheng, Y.; Meng, R.; Tan, C.; Chen, X.; Xiao, J. Selective gas adsorption and I-V response of monolayer boron phosphide introduced by dopants: A first-principle study. Appl. Surf. Sci. 2018, 427, 176-188.

4 Perdew, J. P.; Burke, K.; Ernzerhof, M. Generalized gradient approximation made simple. Phys. Rev. Lett. 1996, 77, 3865.

5 Sorescu, D. C.; Rice, B. M. Theoretical predictions of energetic molecular crystals at ambient and hydrostatic compression conditions using dispersion corrections to conventional density functionals (DFT-D). J. Phys. Chem. C. 2010, 114, 6734-6748. 